\title{
THE PASSAGE OF TRYPANOSOMA GAMBIENSE THROUGH MUCOUS MEMBRANES AND SKIN.
}

\author{
By EDWARD HINDLe, Ph.D., A.R.C.S., F.L.S., \\ Magdalene College, Cambridge: Beit Memorial Research Fellow.
}

\section{(From the Quick Laboratory, University of Cambridge.)}

Although in nature Sleeping Sickness is chiefly transmitted by Glossina palpalis, the possibility of other modes of infection has been suggested by various authors.

In 1907 Koch brought forward evidence which seemed to show that this disease may be transmitted by coitus, for, in his report from Uganda, the following note occurs: "All attempts to find G. palpalis in that part of Kisiba in which the disease chiefly occurs have been proved unavailing; it may now be assumed to be certain that the fly does not occur in those regions." Here 15 infected women were found. "These had never left Kisiba and could only have been infected there. It is, however, further established that the women are all married, that their husbands either have died of Sleeping Sickness, or are suffering from the disease. Of particular importance for the significance of this occurrence of Sleeping Sickness in married women is that in one case a man with trypanosomiasis has three wives and that all three also suffer in a demonstrable manner from trypanosomiasis; one of them is already very ill. From this it must be concluded that the infection in this case can have been brought about only by sexual intercourse. If any other agency, e.g. bloodsucking insects, were to convey the disease in Kisiba from infected persons to healthy not only women whose husbands suffer from trypanosomiasis must have fallen ill, but also women with healthy husbands, unmarried women, moreover children and older people who live in close contact with sick." (Quoted from Sleeping Sickness Bulletin, I. pp. 72-73.) 
It is the opinion of most investigators, however, that further evidence of such transmission is necessary before we can consider it definitely proved, and with a view to testing its possibility, the writer has performed various experiments to see if $T$. gambiense is capable of passing through a sound mucous membrane and also the undamaged epidermis.

Numerous authors have carried on various feeding experiments with other species of trypanosomes, with somewhat contradictory results. Whereas on the one hand Musgrave and Williamson ${ }^{3}$ carried on feeding experiments with Surra for more than a year, with "hundreds of animals," and obtained no infection, Manteufel', working with T. lewisi, always obtained infection by introducing infected blood into the mouths of rats. The latter author also showed that T. lewisi is capable of passing through the skin of rats and infecting them, but up to the present no similar experiments with $T$. gambiense have been recorded.

The following experiments, therefore, have been performed with a view to ascertaining whether $T$. gambiense behaves in a similar manner to $T$. lewisi as regards passage through mucous membranes and the skin :

(a) Infection per os. A number of experiments were performed by feeding uninfected rats with the organs of animals which had died of Sleeping Sickness. Although five rats were fed in this fashion, none of them became infected, and thus, at first, it appeared as if infection by feeding could not be effected.

A further series of experiments were made, however, and instead of feeding the rats with the organs of animals which had died of trypanosomiasis, 1 c.c. of infected blood was introduced into the mouth of each rat by means of a syringe. Under these conditions the rats always became infected after an incubation period of from 7-10 days. Great care was taken to prevent the possibility of producing lesions in the mouths of the rats used, and there can be no doubt that the trypanosomes made their way into the blood through the mucous membrane of the alimentary canal.

The contradictory results obtained by feeding with the organs of infected animals and infected blood mixed with food, seems to be due to the effect of the digestive fuids on the trypanosomes. When solid matter

1 Musgrave and Williamson, Bulletin No. 3, Bureau of Government Laboratories Manila, P. I.

2 Manteufel, Arb. a. d. Kais. Gesundh. 1909, vol. sxxiIr. pp. 46-83. 
is given to the rats, there will be a more copious secretion of gastric juice and also fewer parasites introduced, than when blood is injected into the mouth, and consequently there will be a greater chance of the trypanosomes being overcome before they have been able to penetrate the wall of the alimentary canal. It was found by experiment that trypanosomes when mixed with gastric juice soon become nonmotile, and the rapidity with which this takes place depends upon the relative proportions of the trypanosome-containing blood and the gastric juice.

(b) Infection per vaginam. A few experiments were made to ascertain whether $T$. gambiense can be transmitted from rat to rat by coitus, but although infected males were kept with healthy females and vice-versî, up to the present we have not obtained any infection by these means. In addition a number of female rats were isolated and into the vagina of each a drop of infected blood was introduced by means of a small and carefully rounded glass tube, great care being taken to avoid the possibility of producing any abrasions. After incubation periods varying from 5-8 days all the rats became infected and the disease followed its normal course. This experiment has been repeated six times, in every case with positive results.

(c) Infection per cutaneam. The following experiment was carried out to ascertain if $T$. gambiense could pass through an undamaged epithelium. A drop of infected blood was spread over some part of the body of a rat, whose hair had neither been shaved nor cut, and allowed to dry. After about one hour the dried blood was covered with a layer of collodion, and the rat then set free. After a normal incubation period three (two males and one female) out of five rats thus treated became infected. On repeating these experiments with rats that had been shaved success was invariable, but in the operation of shaving there is always the possibility of producing small abrasions.

The foregoing experiments clearly show that in rats T. gambiense is able to penetrate a sound mucous membrane and also an undamaged skin and there is no reason to suspect that this parasite would not possess similar powers in its normal host, man. Moreover, the ease with which it passes through the mucous membrane of the vagina, shows that there is every possibility of Sleeping Sickness being transmitted by coitus.

Although there is no direct evidence to show that trypanosomes ever occur in the seminal fluid, yet in the case of dourine (T. equiperdum.) transmission normally takes place by coitus and it is necessary 
to assume, either the presence of lesions in the infected partner, or the occurrence of trypanosomes in the seminal fluid.

The experiments recorded above show that in the case of T. gambiense there is no necessity for any lesion in the mucous membrane of the healthy partner for infection to take place, once the parasites are brought in contact with it. In restricting the spread of Sleeping Sickness it is important, therefore, to insist upon the necessity for personal prophylaxis, even though infection other than by Glossina palpalis (and possibly G. morsitans) is probably exceptional.

Note:-Since this paper was written Martin and Ringenbach ${ }^{1}$ have published an account of similar experiments with $T$. gambiense in guinea-pigs. These authors obtained infection by introducing infected blood into the vagina of guinea-pigs and also by rubbing infected blood on the shaved skin. They failed to obtain infection, however, by spreading blood on the unshaven skin, except in the case of two females, in which the writers consider that the infected blood ran down the belly and entered the vagina of each of the animals. The experiments recorded above show that, in the case of rats, $T$. gambiense may pass through the unshaven skin, for the experimental conditions were such that it was impossible for the infected blood to come in contact with any mucous membrane.

1 Bull. Soc. Path. Exot. 1910, pp. 433-437. 